We shall note a few additional properties of the function we have obtained.

In addition to being single valued, $F(x)$ assumes a given value but once. We can thus regard it as giving a one-to-one transformation of the interval $(0,1)$ into itself, which is everywhere discontinuous. At every point save $x=\frac{1}{2}$ the function has no limit; that is, every point, except $x=\frac{1}{2}$, is a point of discontinuity of the second kind. It is also apparent that both the greatest and least values approached at a point are continuous functions.

INDIANA UNIVERSITY, May, 1914.

\title{
PROOF OF THE CONVERGENCE OF POISSON'S INTEGRAL FOR NON-ABSOLUTELY INTEGRABLE FUNCTIONS.
}

BY DR. W. W. KÜSTERMANN.

IN the following pages I propose to give a proof of the

Theorem: If $f(x)$ is a real, periodic function, of period $2 \pi$, which in the interval $(0,2 \pi)$ has a proper or improper integral in the sense of Lebesgue, Harnack-Riemann, or HarnackLebesgue-Hobson, ${ }^{*}$ then

$$
\begin{aligned}
& \lim _{r \rightarrow 1} \frac{1}{2 \pi} \int_{-\pi}^{\pi} f(\alpha) \frac{1-r^{2}}{1+r^{2}-2 r \cos (\alpha-x)} d \alpha \\
& \quad=\lim _{t \rightarrow 0} \frac{1}{2}[f(x+t)+f(x-t)]
\end{aligned}
$$

at every point $x$ where the limit on the right hand side exists.

This theorem includes in particular the case where $f(x)$ remains finite-disposed of by Schwarz, $\dagger$ and the case where $f(x)$ becomes infinite at an infinite number of points, but has an absolutely convergent improper integral-discussed by Hobson and others. $\neq$ Moreover, it goes farther, in that it

* For these definitions see Hobson, Theory of Functions of a Real Variable, Cambridge, 1907.

† Schwarz, Math. Abhd., vol. 2, pp. 144 and 175.

$\$$ Most completely by Hobson, Theory of Functions of a Real Variable, p. 719; cf. also Bôcher, Ann. of Math., 2d ser., vol. 7, p. 81; Fatou, Acta Math., vol. 30, p. 335; Picard, Traité d'Analyse, 2 d ed., vol. 1, p. 268; Forsyth, Theory of Functions:, 2d ed., p. 450. 
also covers the case where $f(x)$ becomes infinite in such a way as to be not absolutely integrable in the vicinity of a point, or, for that matter, of a non-dense, closed set of points of content zero. All former proofs, as far as I know, fail to cover this case, since they require at some place or other that $\left|\int f(\alpha) d \alpha\right|$ be replaced by $f \mid f(\alpha) d \alpha$. The subsequent proof rests upon an application of the second mean value theorem, established by Hobson $\dagger$ in its most general form. For the present purpose Hobson's results may be stated as follows:

"If $\varphi(x)$ be a limited and monotonically decreasing positive function of the real variable $x$, and if $f(x)$ have an improper integral in $(a, b)$, either absolutely or non-absolutely convergent in accordance with the definitions of Lebesgue, Harnack-Riemann, or Harnack-Lebesgue-Hobson, then

(I) $\varphi(x) f(x)$ has also an improper integral in $(a, b)$ in accordance with the same definition;

$$
\text { (II) } \int_{a}^{b} \varphi(x) f(x) d x=A \int_{a}^{x} f(x) d x \text {, where } A \text { is any number }
$$

such that $A \geqq \varphi(a+0)$ and $X$ is a number in the interval $(a, b) . "$

Now consider

$$
\varphi_{r}(\alpha)=\frac{1-r^{2}}{1+r^{2}-2 r \cos \alpha},
$$

where $0<r<1$ in the interval $0 \leqq \alpha \leqq \pi$. The $\cos \alpha$ decreases monotonically from +1 to -1 . The expression $1+r^{2}-2 r \cos \alpha$, however, increases from $(1-r)^{2}$ to $(1+r)^{2}$ and remains always greater than zero. It follows that, for each $0<r<1, \varphi_{r}(\alpha)$ is a positive, monotonically decreasing function and satisfies the premises of the mean value theorem. If then $f(x)$ does likewise, we may conclude from (I) that Poisson's integral

$$
P(x, r)=\frac{1}{2 \pi} \int_{-\pi}^{\pi} f(\alpha) \frac{1-r^{2}}{1+r^{2}-2 r \cos (\alpha-x)} d \alpha
$$

* Thus the presence of just one singularity such as $\frac{\sin 1 / \alpha}{\alpha}$ has at $\alpha=0$ would question the existence of Poisson's integral and its limit for any point $x$ whatever.

† Hobson, Proc. Lond. Math. Soc., 2d ser., vol. 7 (1909), p. 14. 


$$
\begin{aligned}
& =\frac{1}{2 \pi} \int_{-\pi}^{\pi} f(x+\alpha) \frac{1-r^{2}}{1+r^{2}-2 r \cos \alpha} d \alpha \\
& =\frac{1}{2 \pi} \int_{-\pi}^{\pi} f(x+\alpha) \varphi_{r}(\alpha) d \alpha
\end{aligned}
$$

exists and has meaning. Now

$$
\int \frac{\left(1-r^{2}\right) d \alpha}{1+r^{2}-2 r \cos \alpha}=\arctan \left[\frac{1+r}{1-r} \tan \frac{\alpha}{2}\right],
$$

hence

$$
\int_{-\pi}^{\pi} \varphi_{r}(\alpha) d \alpha=2 \pi
$$

independent of $r$. Let

$$
\lim _{t \rightarrow 0} \frac{1}{2}[f(x+t)+f(x-t)]=L,
$$

then

$$
\begin{aligned}
& 2 \pi\{P(x, r)-L\}=\int_{-\pi}^{\pi}[f(x+\alpha)-L] \varphi_{r}(\alpha) d \alpha \\
&=2 \int_{0}^{\pi}\left[\frac{f(x+\alpha)+f(x-\alpha)}{2}-L\right] \varphi_{r}(\alpha) d \alpha, \\
&=2\left\{\int_{0}^{\delta}+\int_{\delta}^{\pi}\right\}\left[\frac{f(x+\alpha)+f(x-\alpha)}{2}-\varphi_{r}(-\alpha),\right. \\
& \text { since } \varphi_{r}(\alpha)=\varphi_{r}(\alpha) d \alpha .
\end{aligned}
$$

An arbitrarily small $\epsilon>0$ having been prescribed, let $\delta$ be chosen in such a way that

$$
\left|\frac{f(x+\alpha)+f(x-\alpha)}{2}-L\right|<\epsilon \text {, for } 0<\alpha<\delta .
$$

That this is always possible follows from (1). Moreover, $\varphi_{r}(\alpha)$ being positive, $\left|\varphi_{r}(\alpha)\right|=\varphi_{r}(\alpha)$. Hence, applying the second mean value theorem (II) to $\int_{\delta}^{\pi}$, we have 


$$
\begin{aligned}
& \pi|P(x, r)-L| \leqq \epsilon \int_{0}^{\delta} \varphi_{r}(\alpha) d \alpha \\
&+\varphi_{r}(\delta) \cdot\left|\int_{\delta}^{X_{r}}\left[\frac{f(x+\alpha)+f(x-\alpha)}{2}-L\right] d \alpha\right|, \\
&<\epsilon \pi+\varphi_{r}(\delta) G, * \quad \text { where } \delta \leqq X_{r} \leqq \pi, \\
&<\epsilon \pi+\frac{\left(1-r^{2}\right) K}{\sin ^{2} \frac{1}{2} \delta} \\
& \quad \text { where } G \text { and } K \text { are positive constants, } 0<<1, \\
&<\epsilon^{\prime}, \quad
\end{aligned}
$$

for $r$ close enough to unity. Hence $\lim _{r \rightarrow 1} P(x, r)=L$.

ANn Arbor,

W. W. KüSTERMANN.

May 2, 1914.

\section{THE NAPIER TERCENTENARY CELEBRATION.}

The Napier Tercentenary Celebration was held at Edinburgh on July 24-28, 1914, with over three hundred visitors present. The ceremonies opened at the University of Edinburgh under the presidency of the Lord Provost of the city, the address of the day being delivered by Lord Moulton and relating to the probable reasons in Napier's mind for deciding upon a table of logarithms of sines and to his probable methods of computing. Brief addresses were given by four of the official delegates, Professors Andoyer and D'Ocagne of Paris, Smith of New York, and Bauschinger of Strassburg.

On Saturday morning the first session was held under the presidency of Professor Hobson of Cambridge. The following papers were presented:

Dr. J. W. L. Glatsher, of Cambridge: "The work of Napier."

Dr. Glaisher called attention to the paucity of notations

* The argument here is based on the fact that a definite integral is a continuous function of its upper limit and as such has a finite maximum and minimum. Cf. E. H. Moore, Transactions, vol. 2, pp. 296 and 459. 